\title{
Medical emergencies in medical imaging
}

\author{
John A Staples, ${ }^{1,2}$ Donald A Redelmeier ${ }^{1,2,3,4}$
}

Sending inpatients to the medical imaging department is sometimes tantamount to discharging them from hospital for hours at a time. Consider, for example, a patient with an unexplained acute abdomen where an urgent CT scan is indicated. Patient transport, logistical delays and the procedure itself may lead to gaps in monitoring vital signs, providing intravenous fluids and administering medications (eg, antibiotics, antianginals and analgesics). For stable patients, even basic tasks such as eating, toileting, physical therapy, family meetings and discharge planning can be problematic while undergoing medical imaging. Ironically, the gaps in general medical care for inpatients in a medical imaging department may occur in full view of healthy outpatients awaiting elective imaging procedures.

This issue of the journal contains a descriptive study by Ott and colleagues that highlights how medical emergencies in medical imaging departments are neither rare nor benign. ${ }^{1}$ The study examined lifethreatening changes in patient status occurring in the medical imaging department of one large American hospital over a 2-year period. The

\footnotetext{
${ }^{1}$ Institute for Clinical Evaluative Sciences, Toronto, Ontario, Canada; ${ }^{2}$ Division of General Internal Medicine, Sunnybrook Health Sciences Centre, Toronto, Ontario, Canada; ${ }^{3}$ Clinical Epidemiology Program, Sunnybrook Research Institute, Toronto, Ontario, Canada; ${ }^{4}$ Centre for Leading Injury Prevention Practice Education \& Research, Toronto, Ontario, Canada
}

Correspondence to Dr Donald A Redelmeier, Sunnybrook Health Sciences Centre, G-151, 2075 Bayview Ave, Toronto, ON M4N 3M5, Canada; dar@ices.on.ca overall frequency averaged about one event per week. Forty per cent of patients originated from critical care wards and about half of the events occurred on the patient's first day of admission. No single physiologic change was responsible for more than a quarter of the emergency events, with hypoxaemia or hypotension as the two most common of the eleven specific triggers examined. About one in four patients died in hospital, but not usually in the medical imaging department.

The study offers a careful discussion that appropriately avoids causal attributions. One interpretation is that seriously ill patients are both prone to undergoing complex imaging procedures and already predisposed to subsequent complications. An alternative interpretation is that medical care for inpatients in the medical imaging department is unable to prevent serious medical instability. Many other interpretations are possible depending on unmeasured metrics such as the duration of patient stay in the medical imaging department, the gymnastics involved in positioning for each procedure and the medications used to optimise image quality. None of these interpretations would justify a complacent attitude towards hospital inpatients in the medical imaging department.

Ott and colleagues provide some pithy details for clinicians seeking to understand and anticipate these emergencies. In particular, about two-thirds of patients were receiving respiratory support (most commonly supplemental oxygen) and at least a quarter of patients received sedative medications (benzodiazepines, opioids, propofol or paralytics) around the time of medical imaging. Although the study did not explore the amount of overlap between these two groups, at least some emergencies may be related to the administration of respiratory depressants to patients with pre-existing respiratory compromise. This complication can be avoided.

Another nuanced observation made by Ott and colleagues is that few patients showed major abnormalities in vital signs to warn clinicians of the upcoming emergency. The seemingly abrupt pace of deterioration may represent the natural progression of disease, the effect of the imaging procedure or a failure by hospital staff to perform appropriate vital sign surveillance. Another possibility is that the logistical challenges of organising an imaging procedure may distract from patient assessment and dynamic re-evaluation of the appropriateness of proceeding as originally planned. None of these explanations is an argument against monitoring the patient's vital signs and, indeed, may suggest that vital signs need to be monitored more frequently and carefully in the medical imaging department.

Emergencies in medical imaging can also lead to finding fault with healthcare professionals. Confronted with a deteriorating patient, some ward physicians may have ordered imaging studies prior to conducting a clinical assessment or a more thoughtful evaluation. Some overburdened ward nurses may have subconsciously benefited from the transportation of the patient off the ward for hours. Some radiology technicians may have focused on the production of high-quality images rather than the greater goal of getting the patient better. The frequency of individual error is unclear; however, transitions between the ward and the imaging department inevitably create an environment for diffusion of responsibility, ${ }^{2}$ 
fumbled handoffs ${ }^{3}$ and the blaming of other healthcare professionals.

A number of psychological forces may further influence the frequency with which medical emergencies occur in the medical imaging department. In particular, the production and interpretation of images can be highly lucrative, creating a financial conflictof-interest that may cause the benefits of imaging to be exaggerated and its harms minimised. ${ }^{4}$ Once captured, images are readily digitised and available for remote viewing, potentially encouraging clinicians to perceive the patient as a computer icon rather than as a human being. Indeed, the subsequent images can seem metaphysically more glamorous and sanitised than patients themselves. These psychological influences are difficult to study in a scientific manner and their influence on day-to-day medical decision making remains untested.

The emergencies in medical imaging reinforce other cautions against excessive diagnostic technology. The maxim of 'do no harm' implies that physicians must always remain wary of iatrogenic complications such as nephrotoxicity from intravenous radiocontrast agents, aspiration pneumonia from excessive sedation, nosocomial infections from surface contaminants and malignancies attributable to the effect of ionising radiation. ${ }^{5} 6$ Principles of cost-effectiveness argue that diagnostic technology should be used to improve patient outcomes rather than to satisfy health professionals' curiosity. ${ }^{7-9}$ Finally, evidence-based medicine suggests that the adverse events related to false-positive and false-negative test results need to be carefully considered even if a technology is safe and informative for anecdotal cases. ${ }^{10}$

Clinicians should continue to send selected inpatients for medical imaging when the benefits of a correct diagnosis exceed the risks of the procedure. Nevertheless, the study by Ott and colleagues reminds us that the risks associated with medical imaging for hospital inpatients extend beyond the risk of the procedure itself. The data might also motivate more creative solutions such as specialised transport teams for patients recognised to be at risk of adverse events, ${ }^{11}$ dedicated nursing for patients in medical imaging departments, protocols specifying appropriate frequencies of vital sign monitoring and innovations to patient transport that reduce time away from the ward. In addition, an advance orientation might be warranted for emergency response teams around the layout of a complex medical imaging department. The medical imaging department is hardly the right place to run a resuscitation.

\section{Competing interests None.}

Provenance and peer review Commissioned; internally peer reviewed.
Published Online First 23 March 2012

BMJ Qual Saf 2012;21:446-447.

doi:10.1136/bmjqs-2012-000817

\section{REFERENCES}

1. Ott LK, Pinsky MR, Hoffman LA, et al. Medical emergency team calls in the radiology department: patient characteristics and outcomes. BMJ Qual Saf (2012). doi:10.1136/bmjqs-2011-000423

2. Latane B, Williams K, Harkins S. Many hands make light work: the causes and consequences of social loafing. J Personality Soc Psychol 1979;37:822-32.

3. Ong M, Coiera E. A systematic review of failures in handoff communication during intrahospital transfers. Jt Comm J Qual Patient Saf 2011;37:274-84.

4. Jørgensen KJ, Klahn A, Gøtzsche PC. Are benefits and harms in mammography screening given equal attention in scientific articles? A cross sectional-study. BMC Med 2007;5:12.

5. Smith-Bindman R, Lipson J, Marcus R, et al Radiation dose associated with common computed tomography examinations and the associated lifetime attributable risk of cancer. Arch Intern Med 2009;169:2078-86.

6. Fazel R, Krumholz HM, Wang $\mathrm{Y}$, et al. Exposure to low-dose ionizing radiation from medical imaging procedures. $N$ Engl $J$ Med 2009;361:849-57.

7. Young L, Wackers FJ, Chyun DA, et al. Cardiac outcomes after screening for asymptomatic coronary artery disease in patients with type 2 diabetes: the DIAD study: a randomized controlled trial. JAMA 2009;301:1547-55.

8. Pines JM. Trends in the rates of radiography use and important diagnoses in emergency department patients with abdominal pain. Med Care 2009;47:782-6.

9. Benarroch-Gampel J, Boyd CA, Sheffield $\mathrm{KM}$, et al. Overuse of CT in patients with complicated gallstone disease. J Am Coll Surg 2011;213:524-30.

10. Wiener RS, Schwartz LM, Woloshin S. Time trends in pulmonary embolism in the United States: evidence of overdiagnosis. Arch Intern Med 2011;171:831-7.

11. Kue R, Brown P, Ness C, et al. Adverse clinical events during intrahospital transport by a specialized team: a preliminary report. Am J Crit Care 2011;20:153-61. 